\title{
Disreflexia autonómica: um desafio diagnóstico
}

Carolina Martins Moreira, ${ }^{1}$ Luís Afonso Vouga, ${ }^{1}$ Maria João Andrade ${ }^{2}$

\section{RESUMO}

A disreflexia autonómica (DA) é uma complicação frequente nas lesões medulares (LM), com risco potencial de vida.A sua apresentação súbita e exuberante, com quadros de hipertensão arterial, bradicardia, fibrilhação auricular, cefaleias, midríase e sudorese profusa leva a que estes doentes sejam encaminhados para o serviço de atendimento urgente mais próximo, onde os profissionais de saúde têm reduzida expe-riência com esta condição.

Este caso clínico retrata o caso de um doente com LM crónica, admitido no serviço de urgência com quadro de DA, que conduziu a internamento e realização exaustiva e desnecessária de exames complementares pela ausência de diagnóstico e orientação atempados.

Com o aumento da prevalência e esperança média de vida desta população, é urgente o reconhecimento desta síndroma clínica potencialmente fatal por parte dos médicos que realizam atendimento em serviços de urgência ou cuidados de saúde primários.

Palavras-chave: Lesão medular; Disreflexia autonómica.

\section{INTRODUÇÃO}

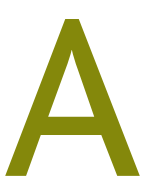

lesão medular (LM) traumática é um evento súbito com um efeito devastador, físico e psicológico para o doente, confrontando o médico com múltiplos desafios na sua gestão, quer na fase aguda quer na fase crónica. ${ }^{1}$

Estima-se que atualmente a incidência de LM traumáticas seja de 40 casos por milhão de habitantes nos Estados Unidos da América e 16 por milhão na Europa Ocidental. ${ }^{2}$ Em Portugal não há dados epidemiológicos concretos relativos a esta patologia mas, num estudo não publicado, encontramos uma incidência de 10 a 15 por milhão de habitantes na população do norte do país e que é reencaminhada para o Hospital Geral de Santo António.

Com a melhoria dos cuidados de saúde e o aumento da esperança média de vida, a prevalência de lesionados medulares em fase crónica está certamente a aumentar. Assim, é premente um conhecimento seguro desta patologia, não só pelos especialistas em medici-

1. Médico Interno de Medicina Física e de Reabilitação. Centro Hospitalar e Universitário do Porto.

3. Médica Assistente de Medicina Física e de Reabilitação. Chefe de Serviço, Responsável pela Unidade de Lesionados Medulares, do Centro Hospitalar e Universitário do Porto. na física e de reabilitação que contactam mais frequentemente com estes doentes, como também pelos clínicos que asseguram a prestação de cuidados médicos em serviços ou consultas de urgência a que estes doentes recorrem em situação aguda.

De entre as complicações mais frequentes na LM crónica que motivam o recurso ao serviço de urgência (SU), excluindo complicações genito-urinárias e respiratórias, destaca-se a disreflexia autonómica (DA) pelo seu caráter súbito com potencial risco de vida, podendo ocorrer em qualquer LM (completa ou incompleta) com nível neurológico acima de T6, havendo mesmo relatos na literatura de casos com lesões até T10. ${ }^{3}$

Um episódio de DA é secundário a qualquer estímulo nóxico abaixo do nível da lesão. Em $85 \%$ dos casos é de origem urológica por infeção do trato urinário ou distensão vesical, muitas vezes provocada por obstrução da algalia. ${ }^{4}$ É caracterizado por uma súbita elevação da tensão arterial (TA) sistólica de 20 a 30mmHg, ${ }^{5-6}$ normalmente associada a cefaleia. Tendo em conta que a TA basal em doentes com LM cervical ou torácica alta é habitualmente 15 a 20mmHg inferior à de um indivíduo sem lesão, valores tidos como «normais» para a população geral poderão indicar já um estado de DA nestes doentes. 
Concomitantemente, poderão estar presentes diversos sintomas: bradicardia, fibrilhação auricular, cefaleias, rubor facial, midríase, sudorese profusa, congestão nasal e sensação de morte iminente. ${ }^{7} \mathrm{O}$ quadro poderá variar desde um leve desconforto até uma situação de emergência, com risco de hemorragia intracraniana, descolamento da retina e morte. ${ }^{6}$

Apesar de a DA ser uma entidade classicamente confinada à fase crónica da LM, a presença de DA na fase aguda da LM encontra-se já reportada na literatura, ${ }^{8}$ pelo que deverá ser reconhecida como um possível diagnóstico diferencial.

A resolução de um quadro de DA obriga a um diagnóstico rápido com imediata instituição de medidas terapêuticas adequadas.

Devido à instalação súbita de sintomas, frequentemente severos, estes doentes são muitas vezes encaminhados para o serviço de atendimento urgente mais próximo, ${ }^{3}$ onde poderão ser observados por clínicos com limitada experiência neste tipo de patologia, conduzindo necessariamente a um atraso na identificação do quadro, com potenciais repercussões prognósticas, como já demonstrado na realidade nacional. ${ }^{9}$

O caso que se relata trata de um cenário típico de DA, em que o atraso no diagnóstico levou ao prolongamento de tempo de internamento, bem como à realização de inúmeros exames auxiliares de diagnóstico (EAD) desnecessários.

\section{DESCRIÇÃO CLÍNICA}

Homem, 48 anos, caucasiano, tetraplegia American Spinal Injury Association Impairment Scale (AIS) B, nível neurológico C6, sequela de acidente de viação em 1988, casado, com bom suporte familiar, profissionalmente ativo, funcionário administrativo na função pública. Previamente seguido em consulta de fisiatria, lesões medulares, tendo perdido o seguimento por faltas consecutivas em 2015 motivadas por internamentos e esquecimento por parte do doente.

Recorre ao SU de um hospital central em 20/07/2017 em contexto de infeção urinária resistente a antibioterapia instituída em ambulatório (amoxicilina - ácido clavulânico e furadantina), mantendo picos febris e tendo já urocultura a documentar a presença de um enterococcus faecalis. Mantinha regime miccional prévio com micção reflexa e perdas para coletor.
No SU realiza ecografia renal e prostática, bem como Rx cervical (por história de queda semanas antes), sem alterações de novo. Analiticamente apresentava neutrofilia relativa e PCR de 171 (valores de referência $0-5 \mathrm{mg} / \mathrm{L}$ ).

É assumida pielonefrite aguda, com retenção urinária pelo que foi algaliado e iniciou ceftriaxona.

Após três horas, aquando da passagem do decúbito para sedestação, inicia quadro hipertensivo (TAS $>200 \mathrm{mmHg}$ ) com bradicardia, hipersudorese e tremor. Esta clínica era sistematicamente despertada aquando de levantes para a posição sentada, revertendo após nova passagem para decúbito.

Por este motivo, é mantido em observação no SU nas 48 horas seguintes, com parâmetros inflamatórios analíticos em decrescendo.

Pela manutenção de episódios hipertensivos, bradicardia e tremor associados a levante, é pedida TC Cervical com imagens sobreponíveis ao exame anterior de 2011 (artrodese C3-C7, com canal raquidiano amplo).

É decidido internamento em medicina interna para estudo filiado nos episódios descritos, no sentido da exclusão de infeção e efeitos laterais ou privação de fármacos, mantendo o doente sempre a clínica descrita. Realiza ecografia abdominal e estudo analítico com ionograma, estudo da função tiroideia, hepática, enzimas cardíacas, sem alterações de relevo. É pedida TAC toraco-abdomino-pélvica, sem alterações de relevo.

Ao sétimo dia de internamento é solicitada colaboração da medicina física e de reabilitação. Nesta observação, exame objetivo sobreponível com o último descrito em consulta, sem agravamento da espasticidade, novas lesões cutâneas, ungueais ou da mucosa.

Revendo a história com o doente e após consulta do processo clínico, havia registo anterior de eventos similares, nomeadamente aquando da realização de estudo urodinâmico (manipulação genital, introdução de sonda) e igualmente em situação de repleção vesical, obstipação ou vestuário apertado, sendo que nestes casos não houve necessidade de recorrer aos cuidados de saúde.

O diagnóstico de DA foi colocado como mais provável, pelo facto de os sintomas serem suscitados invariavelmente pela mudança de posição (decúbito para posição sentada), sendo favorecida a hipótese de causa mecânica, neste caso pela presença de algália a 
causar possível compressão da parede vesical/uretral desencadeada pela sedestação.

Como o doente urinava previamente por manobras e tinha já completado uma semana de tratamento antibiótico dirigido procedeu-se à desalgaliação, com reversão da sintomatologia.

Ficou $48 \mathrm{~h}$ sob vigilância, mantendo micção por perdas para coletor e com medição de volumes residuais seriados (valores consistentemente inferiores a $100 \mathrm{ml}$ ).

Não teve novos episódios de DA, pelo que lhe foi dada alta para o domicílio e reencaminhado para a consulta externa de lesões medulares.

Posteriormente à observação nessa consulta, 49 dias mais tarde, apresentou novo episódio de DA, com TA 184/107 em sedestação que, pela sua identificação imediata, se resolveu com mudança postural (para decúbito) e evicção de potenciais estímulos nóxicos externos. Ao exame objetivo apresentava lesão isquiática direita com drenagem e com endurecimento cutâneo. Foi proposto internamento urgente para estudo, após o qual se assumiu que na base deste novo episódio de disreflexia estaria a etiologia mecânica por compressão e falta de «almofadamento» natural da região isquiática (massa cicatricial e perda de tecidos moles), agravada pela presença de úlcera de pressão isquiática.

Após evolução favorável do quadro teve alta com novo sistema (almofada) antiescara e com indicação para realizar mudanças posturais frequentes para evitar o desenvolvimento de novas úlceras de pressão.

\section{COMENTÁRIO}

Entre as disfunções autonómicas que ocorrem após lesão medular, numa fase crónica, a DA surge na literatura como uma das menos conhecidas pelos médicos que não trabalham habitualmente com LM. ${ }^{9}$

A DA consiste numa constelação de sinais e sintomas que ocorrem em lesionados medulares com nível neurológico superior ou igual a $\mathrm{T} 6$, sendo tanto mais frequente quanto mais alta for a lesão. ${ }^{6-7}$ É consequência da interrupção do controlo supraespinhal sobre o sistema nervoso simpático (SNS), provocando uma ativação massiva, reflexa e desregulada do mesmo, na ausência de uma resposta capaz por parte do sistema parassimpático. Isto ocorre porque as vias aferentes que conduzem os estímulos periféricos ou viscerais aos neurónios localizados nos núcleos intermédio medial e lateral toracolombares se mantêm íntegras. Consequentemente, ocorre ativação simpática reflexa e libertação de dopamina e noradrenalina que conduzem à mobilização do leito vascular esplâncnico e vasoconstrição periférica com aumento da TA. ${ }^{7}$ Este mecanismo vai desencadear uma resposta por parte dos barorrecetores aórticos e carotídeos, bem como dos centros vasomotores do tronco cerebral, resultando no aumento da atividade parassimpática e nos sintomas classicamente descritos na DA, explicados pelo predomínio simpático abaixo e parassimpático acima do nível de lesão. ${ }^{10}$

A sintomatologia permanecerá até o estímulo ser retirado, ${ }^{11}$ pelo que facilmente se depreende que um atraso no diagnóstico e tratamento poderá resultar em complicações como o acidente vascular cerebral, convulsões e morte. Por outro lado, episódios de DA podem ocorrer múltiplas vezes ao longo do dia devido a estímulos aferentes não identificados ou não controlados, prejudicando a qualidade de vida destes doentes, de tal modo que não é surpreendente que um estudo em larga a escala ${ }^{12}$ tenha reportado que, na fase crónica, quer os tetraplégicos quer os paraplégicos priorizam a eliminação destes episódios face à possibilidade de readquirirem capacidade de marcha. ${ }^{13}$

O tratamento da DA consiste nas medidas e intervenções estabelecidas nas guidelines clínicas do Consortium for Spinal Cord Medicine, devendo ser executadas sistematicamente e respeitado o algoritmo nele divulgado: ${ }^{13}$

1. Verificar a TA do indivíduo. Se elevada, e o doente se encontrar em posição supina, de imediato sentar o doente.

2. Remover qualquer vestuário, calçado, meias elásticas ou outro dispositivo que possa estar a causar compressão.

3. Monitorizar TA e frequência cardíaca (FC) a cada 5 minutos.

4. Iniciar pesquisa de estímulo desencadeador, iniciando pelo sistema genito-urinário.

5. Se doente algaliado, verificar sistema para possível obstrução ou proceder a substituição de algalia. Se doente não algaliado, proceder à sua algaliação (previamente inserir lidocaína em gel $2 \%$ na uretra e aguardar alguns minutos).

6. Se após estas manobras a TA permanecer elevada, 
medicar com anti-hipertensor de curta duração de ação e pesquisar possível impactação fecal com utilização de lubrificante e anestésico tópico (lidocaína $2 \%$ ).

7. Caso o quadro se resolva, manter vigilância da TA e sintomas durante 2 horas. No caso de se manter quadro de DA (com TA sistólica igual ou superior a $150 \mathrm{mmHg}$ ) e não se identificar uma causa, orientar doente para urgência hospitalar onde deverá ter atendimento urgente para controlo tensional e investigação diagnóstica.

Todo o episódio deverá ficar documentado, desde sintomas na apresentação, resposta clínica, medidas conservadoras e farmacológicas (se aplicável), bem como a investigação diagnóstica. Na maioria dos casos é possível identificar o estímulo nóxico e, ao removê-lo, resolver a DA sem recurso a medicação.

Com o aumento da esperança média de vida nos lesionados medulares, a possibilidade de um doente com este quadro recorrer a uma consulta urgente em cuidados de saúde primários é cada vez maior, pelo que os especialistas em medicina geral e familiar deverão estar familiarizados com este diagnóstico, uma vez que se poderá tratar de uma situação emergente com risco de vida para estes doentes e cuja resolução assenta, primariamente, na identificação e correção do estímulo nóxico.

\section{REFERÊNCIAS BIBLIOGRÁFICAS}

1. DeLisa JA, Gans BM, Walsh NE, editors. Physical medicine and rehabilitation: principles and practice. 4th ed. Philadelphia: Lippincott Williams \& Wilkins; 2005. ISBN 9780781741309

2. Lee BB, Cripps RA, Fitzharris M, Wing PC. The global map for traumatic spinal cord injury epidemiology: update 2011, global incidence rate. Spinal Cord. 2014;52(2):110-6.

3. Consortium for Spinal Cord Medicine. Acute management of autonomic dysreflexia: individuals with spinal cord injury presenting to healthcare facilities. J Spinal Cord Med. 2002;25 Suppl 1:S68-88.
4. Allen KJ, Leslie SW. Autonomic dysreflexia. Treasure Island: StatPearls Publishing; 2019.

5. Teasel RW, Arnold JM, Krassioukov A, Delaney GA. Cardiovascular consequences of loss of supraspinal control of the sympathetic nervous system after spinal cord injury. Arch Phys Med Rehabil. 2000;81(4): 506-16.

6. Krassioukov A, Warburton DE, Teasell R, Eng JJ. A systematic review of the management of autonomic dysreflexia after spinal cord injury. Arch Phys Med Rehabil. 2009;90(4):682-95.

7. Roque V, Cunha I, Rocha A, Andrade MJ. Disfunções autonómicas após lesão medular [Autonomic nervous system dysfunction after spinal cord injury]. Rev Soc Port Med Fis Reabil. 2013;24(2):43-51. Portuguese

8. Krassioukov AV, Furlan JC, Fehlings MG. Autonomic dysreflexia in acute spinal cord injury: an under-recognized clinical entity. J Neurotrauma. 2003;20(8):707-16.

9. Bettencourt M, Carvalho MP, Faria F. Disreflexia autonómica: o que se sabe nos serviços de urgência em Portugal? [Autonomic dysreflexia: what level of knowledge in Portuguese emergency rooms?]. Rev Soc Port Med Fis Reabil. 2016;(1):10-5. Portuguese

10. Milligan J, Lee J, McMillan C, Klassen H. Autonomic dysreflexia: recognizing a common serious condition in patients with spinal cord injury. Can Fam Physician. 2012;58(8):831-5.

11. Eldahan KC, Rabchevsky AG. Autonomic dysreflexia after spinal cord injury: systemic pathophysiology and methods of management. Auton Neurosci. 2018;209:59-70.

12. Anderson KD. Targeting recovery priorities of the spinal cord injured population. J Neurotrauma. 2004;21(10):1371-83.

13. Autonomic Dysreflexia Guideline Development Panel. Acute management of autonomic dysreflexia: adults with spinal cord injury presenting to health-care facilities - Consortium for spinal cord. J Spinal Cord Med. 1997;20(3):284-308.

\section{CONFLITO DE INTERESSES}

Os autores declaram não ter quaisquer conflitos de interesse.

\section{ENDEREÇO PARA CORRESPONDÊNCIA}

Carolina Martins Moreira

E-mail: carolinamoreira.med@gmail.com

https://orcid.org/0000-0001-8781-1823

Recebido em 02-12-2018

Aceite para publicação em 07-07-2019 


\section{ABSTRACT}

\section{AUTONOMIC DYSREFLEXIA: A CHALLENGING DIAGNOSIS}

Autonomic dysreflexia (AD) is a frequent complication of spinal cord injury $(\mathrm{SCl})$ poten-tially life-threatening. Its sudden and exuberant presentation with arterial hypertension, bradycardia, atrial fibrillation, headache, mydriasis, and profuse sweating leads these patients to the nearest emergency department (ED) where health professionals have limited experience with this condition.

This clinical case portrays a patient with chronic $\mathrm{SCl}$ admitted to the ED with an $\mathrm{AD}$, which leads to hospital admission and subjection to an exhaustive and unnecessary battery of exams due to the lack of diagnosis and guidance.

With the increase in prevalence and average life expectancy of this population, it is paramount that doctors working in emergency departments or primary health care can recognize this potentially fatal clinical syndrome.

Keywords: Spinal cord injuries; Autonomic dysreflexia. 